\title{
The veiling spectrum of DI Cephei and its relationship to emission line profiles
}

\author{
J. F. Gameiro ${ }^{1,2}$, D. F. M. Folha ${ }^{1,3}$, and P. P. Petrov ${ }^{4, \star}$ \\ ${ }^{1}$ Centro de Astrofísica da Universidade do Porto, Rua das Estrelas, 4150-762 Porto, Portugal \\ e-mail: jgameiro@astro.up.pt \\ 2 Departamento de Matemática Aplicada, Faculdade de Ciências da Universidade do Porto, Portugal \\ 3 Instituto Superior da Maia, Av. Carlos de Oliveira Campos, 4475-690 Avioso S. Pedro, Castelo da Maia, Portugal \\ 4 Crimean Astrophysical Observatory, p/o Nauchny, Crimea, 98409, Ukraine
}

Received 6 August 2004 / Accepted 2 September 2005

\begin{abstract}
High spectral resolution variability studies of classical T Tauri stars (CTTS) are an essential instrument for probing the physical conditions and dynamics of their atmospheres and immediate vicinity. The shapes of the excess continuum emission and of the line profiles, their variability and relationship are all crucial tools to achieve that goal. We use high spectral resolution optical data of the CTTS DI Cep to suggest a new diagnostic tool to investigate the relationship between the line emission/absorption and the excess continuum emission. By correlating the veiling continuum to the line flux in discrete velocity bins across the emission line we obtain a correlation profile, from which one can discriminate between parts of the line that relate differently to the veiling. An earlier report of an unexpected hump around $5300 \AA$ in the continuum excess emission spectrum of a couple of CTTS is not explained by current models of those stars. We identified a similar feature in the veiling spectrum of DI Cep and discuss, in this context, the relevance of the broad photospheric absorption features present in the spectra of late-type stars. Regarding DI Cep, we find that its radial velocity seems to be variable but no significant periodicity could be derived, possibly due to inadequate time sampling. We argue that this CTTS is most probably observed nearly equator on. Accretion flows could not be identified directly in the emission lines, but their presence is inferred from the analysis of the veiling spectrum, which yields typical projected accretion rates around $2.5 \times 10^{-7} M_{\odot} \mathrm{yr}^{-1}$.
\end{abstract}

Key words. stars: formation - stars: pre-main sequence - stars: individual: DI Cep - methods: data analysis

\section{Introduction}

T Tauri stars (TTS) represent, as a class, the final stage in the formation of low mass stars. The most active TTS, known as classical TTS (CTTS), are surrounded by a circumstellar accretion disk and are characterised by the presence of strong emission lines and an excess of continuum emission, both strongly variable in time-scales that range from minutes and hours to months and years.

Through the years, the activity displayed by CTTS has been explained as resulting from "deep chromospheres" (Herbig 1962), from interaction with a circumstellar disk through a boundary layer (Lynden-Bell \& Pringle 1974; Bertout et al. 1988), or through a stellar magnetosphere that disrupts the disk a few stellar radii from the photosphere and channels matter from the disk to the stellar surface (Königl 1991; Camenzind 1990). The magnetospheric accretion model, as the latter is known, has been fairly successful in explaining many of the observed properties of CTTS, such as their relatively slow rotation velocities (Shu et al. 1994), the UV and optical excess

* Also Isaac Newton Institute of Chile, Crimean branch. continuum emission (Calvet \& Gullbring 1998), and many characteristics of emission lines, such as the hydrogen lines (Muzerolle et al. 2001).

This success results mainly from comparing model predictions to general observed properties of samples of a few tens of CTTS. However, a detailed and consistent quantitative understanding of even one CTTS system is still far from being achieved. Time variability studies of CTTS using high spectral resolution and a long wavelength coverage is a key tool for better understanding the conditions in the magnetospheres. It allows one both to probe simultaneously accretion shocks through excess continuum emission, winds, and accretion flows through line profile studies of lines requiring different excitation conditions and, crucially, to monitor variations.

DI Cep is a typical CTTS with spectral type G8V-G8IV that displays most of the characteristics of this class of stars (Gahm \& Petrov 1983; Herbig \& Bell 1988). It displays strong emission lines and an excess of continuum emission, both variable in time scales of days (Hessman \& Guenther 1997; Kolotilov et al. 2003). Occasionally short-time scale variability has been observed photometrically (Petrov et al. 1972; Gahm et al. 1977) 
and spectroscopically (Bastian \& Mundt 1979). More recent estimates for the stellar parameters give $R_{\star}=2.5 R_{\odot}$, $L_{\star}=5.1 L_{\odot}$ (Hamann \& Persson 1992), locate it at $300 \mathrm{pc}$ (Kholopov 1959), and give $A_{\mathrm{V}}=0.24-0.9$ (Yu et al. 1986; Hamann \& Persson 1992).

In this work we analyse high spectral-resolution data from DI Cep obtained at various epochs. By covering a large part of its optical spectrum at a resolving power no smaller than 26000 and spanning nearly four years, this data set gives us access to DI Cep's veiling spectrum and line spectra, and gives a chance to study if and how they are related to each other. Although the generally poor time sampling prevents us from drawing strong conclusions on the latter issue, we tackle it in a novel approach. This paper is organised as follows: in Sect. 2 we briefly present details of the observations and data reduction, and in Sect. 3 we capitalise on our ability to measure $v \sin i$ accurately in order to constrain DI Cep's inclination towards the line-of-sight and to roughly estimate its mass and age. In Sect. 4 we present its derived veiling spectrum and interpret it in the context of magnetospheric accretion. Section 5 discusses the presence of an unexpected hump in the veiling spectrum and examines the possibility of originating from systematic effects in the veiling determination, while Sect. 6 presents line variability and reports the likely existence of radial velocity variations. In Sect. 7 we introduce a new diagnostic tool for investigating how veiling changes correlate with changes in discrete wavelength bins in line profiles, and illustrate its use with DI Cep's data set. Finally, in Sect. 8 we close the paper with our conclusions.

\section{Observations and data reduction}

The observations, which roughly span four years, were carried out on three different telescopes: $1.93 \mathrm{~m}$ at the Observatoire de Haute-Provence (OHP), William Herschel Telescope (WHT) and Nordic Optical Telescope (NOT). Table 1 summarises the observation log. The OHP fibre-fed échelle spectrograph ELODIE covered the 3900-6800 $\AA$ region at $R \sim 40000$. Three consecutive spectra were obtained per night, each with an exposure time of one hour. The UES échelle spectrograph at the WHT was used to provide an almost full spectral coverage between 4500 and $9000 \AA$ with $R \approx 45000$. On 1999 October 19 DI Cep was observed during three consecutive hours resulting in 18 spectra, each with an exposure time of $10 \mathrm{~min}$. The NOT échelle spectrograph SOFIN allowed us to obtain spectra with $R \approx 26000$ and to cover the wavelength range between 4800-9000 А.

The data reduction was performed with different software, depending on the instrument where it was acquired: using the INTER-TACOS software provided with the ELODIE spectrograph (Baranne et al. 1996), using IRAF (Tody 1993) for UES data, using the 4A software package (Ilyin 2000) for SOFIN data. Spectra were optimally extracted, and thoriumargon was used for wavelength calibration. All spectra were normalised to the continuum.

The different global efficiencies due to the different telescopes and the instruments used imply that the signal-to-noise ratio (SNR) in the different spectra span a large range (from 25 to 100$)$. When we directly compare spectra obtained with
Table 1. Log of spectroscopic observations. $\mathrm{E}=$ ELODIE, $\mathrm{U}=\mathrm{UES}$ $\mathrm{S}=$ SOFIN. Veiling measurements are at $5600 \AA$ and associated uncertainties.

\begin{tabular}{ccccc}
\hline \hline Date & HJD-2 400000 & Inst. & $\begin{array}{c}\text { Veiling } \\
\text { at 5600 ̊ }\end{array}$ & $\begin{array}{c}\text { Veiling } \\
\text { uncertainty }\end{array}$ \\
\hline 1997 Nov. 8 & 50761.390 & $\mathrm{E}$ & 0.49 & 0.06 \\
1997 Nov. 9 & 50762.351 & $\mathrm{E}$ & 0.52 & 0.07 \\
1997 Nov. 10 & 50763.254 & $\mathrm{E}$ & 0.40 & 0.02 \\
1997 Nov. 12 & 50765.299 & $\mathrm{E}$ & 0.40 & 0.06 \\
\hline 1998 Oct. 8 & 51095.448 & $\mathrm{U}$ & 0.25 & 0.04 \\
1998 Nov. 10 & 51127.592 & $\mathrm{~S}$ & - & - \\
\hline 1999 Jul. 5/6 & 51365.621 & $\mathrm{U}$ & 0.43 & 0.05 \\
1999 Jul. 27 & 51387.595 & $\mathrm{U}$ & 0.27 & 0.03 \\
1999 Jul. 28 & 51388.723 & $\mathrm{U}$ & - & - \\
1999 Oct. 19 & 51471.589 & $\mathrm{U}$ & 0.40 & 0.05 \\
\hline 2001 Jul. 29 & 52120.505 & $\mathrm{~S}$ & 0.46 & 0.09 \\
2001 Jul. 31 & 52121.517 & $\mathrm{~S}$ & 0.35 & 0.05 \\
2001 Aug. 3 & 52124.521 & $\mathrm{~S}$ & 0.34 & 0.07 \\
2001 Aug. 8 & 52129.613 & $\mathrm{~S}$ & 0.28 & 0.05 \\
2001 Aug. 9 & 52130.591 & $\mathrm{~S}$ & 0.38 & 0.08 \\
2001 Aug. 10 & 52131.524 & $\mathrm{~S}$ & 0.43 & 0.09 \\
\hline
\end{tabular}

different instruments/setups, we do it after degrading the resolution to the lowest one observed and after re-sampling the data to the same wavelength bins.

Templates stars were observed on the same nights and with the same setup: HD 75935 (G8V) with ELODIE, HR 7368 (G8V) with UES, and $\sigma$ Dra (K0V) with SOFIN.

\section{Inclination to the line of sight}

The acquired data allowed us to measure DI Cep's $v \sin i$ fairly accurately. Combining that measurement with DI Cep properties available in the literature allowed us to estimate its inclination $i$ to the line of sight. We measured $v \sin i=20.2 \pm$ $1.5 \mathrm{~km} \mathrm{~s}^{-1}$, which combined with the reported 9.2 days rotation period (Kolotilov et al. 2003), implies $R_{\star} \sin i=3.5-4.0 R_{\odot}$. An independent estimate of the stellar radius can be obtained by considering that DI Cep is classified as a G8 IV-V star, i.e $T_{\text {eff }}=5000-5400 \mathrm{~K}(\log (T)=3.70-3.73)$, and that $L_{\star}=5.1 L_{\odot}$ (with a typical error in bolometric luminosity of 0.2 dex), which together yield $R_{\star}=2.0-3.7 R_{\odot}$. The projected radius estimated above is consistent with the upper limit of $R_{\star}$, requiring that $\sin i \approx 1$ and, hence, that DI Cep be seen nearly equator on.

We further note that by placing DI Cep on the pre-main sequence evolutionary tracks by D’Antona \& Mazzitelli (1994) and by Palla \& Stahler (1999), we estimate its mass and age to be $M_{\star}=1.5-2.0 M_{\odot}$ and $t=1-5 \mathrm{Myr}$, respectively.

\section{The veiling spectrum}

The spectrum of DI Cep is characterised by the presence of an excess continuum emission, as well as various emission lines, the most prominent being those of the hydrogen Balmer series and CaII infrared triplet. Weaker emission is also present from the permitted lines of FeII, HeI , NaI D, OI, and from the forbidden lines of [OI] ( $\lambda 6300$ and $\lambda 6363 \AA$ ). 


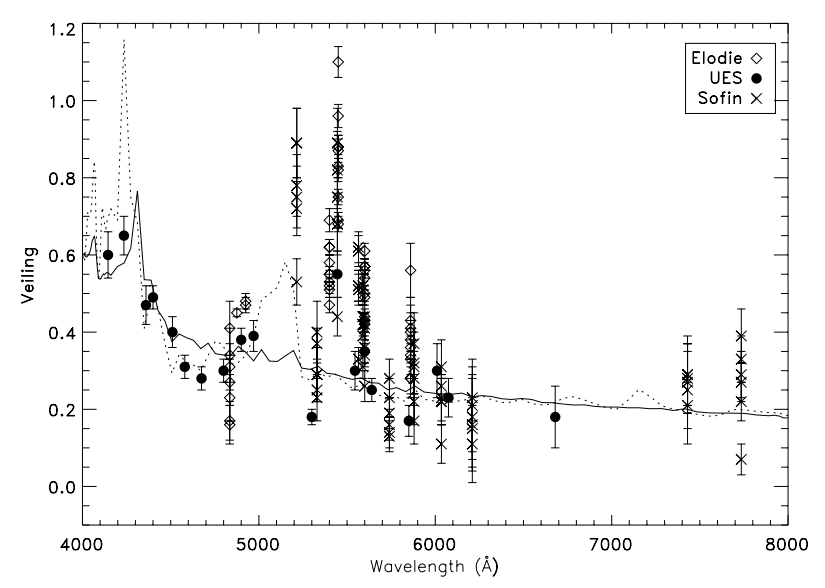

Fig. 1. Veiling measurements. The solid and dotted lines correspond to accretion shock models with: $\log \mathcal{F}=12.0$ and $f=0.024$ on a G8V photosphere; $\log \mathcal{F}=10.5$ and $f=0.06$ on a K7V photosphere (see text).

When we sampled short time-scales (from minutes to hours), we did not find any significant variability in the continuum excess or in emission lines but detected variations on timescales of days and longer. Given the lack of variability observed during single nights, and in order to increase the signal-to-noise ratio of the observed spectra, we averaged the data obtained on the same night.

The non-photospheric excess continuum in DI Cep was analysed through the veiling, which is defined as the ratio between the excess continuum flux and the photospheric continuum's contribution to the spectrum.

The veiling of DI Cep was computed by comparing its spectra with that of HR 7368 after degrading it and re-sampling it to the appropriate spectral resolution and spectral bins. HR 7368, a G8V star, is a good match to DI Cep's spectral type and luminosity class. We tested whether luminosity class III templates could provide a better match to DI Cep photospheric spectra and got a negative result.

The implemented code determines the best fit of the rotationally broadened veiled spectrum of the template star to the spectrum of DI Cep, so we can compute a constant veiling within wavelength regions of $50 \AA$ wide (as proposed by Hartigan et al. (1989). In Fig. 1 we plot our veiling measurements (spanning nearly four years) as a function of wavelength, i.e. the veiling spectrum. For clarity, only measurements with relative uncertainties less than 30\% are shown in Fig. 1.

In general, the veiling tends to increase from the red to the blue, although in a region centred at $5200 \AA$ and about $500 \AA$ wide, the computed veiling is higher than in nearby regions. This feature, henceforth referred to as the veiling hump, will be analysed in more detail in Sect. 5.

There is a strong relationship between variations in veiling at different wavelengths. To illustrate the typical observed variations, Table 1 lists the veiling measurements at $5600 \AA$. We note that variations on time scales of days or years are similar, and there is no clear dependence of the spread in veiling with wavelength (average standard deviation is 0.08). There is a hint of a larger variation of the veiling within the hump, with standard deviations reaching $0.12-0.15$ at three wavelengths between $5200 \AA$ and $5500 \AA$. However, within the same region, standard deviations of 0.06 are also observed. Whether the veiling displays stronger variations in the hump cannot be significantly established from this dataset. Periodogram analysis of the veiling did not reveal the presence of any significant periodicity.

A general increase in veiling from the red to the blue is expected from the magnetospheric accretion shock models (Calvet \& Gullbring 1998) that assume that the excess continuum emission is caused by hot spot(s) on the stellar photosphere, which in turn result from accretion of matter onto the star. We compared the observed veiling spectrum with the one predicted by the Calvet \& Gullbring (1998) shock models, in which the excess continuum spectrum depends mostly on two parameters: the energy flux $\mathcal{F}$ of the accretion flow and the projected surface coverage of the accretion column $f$.

Spectra of the excess continuum emission predicted by Calvet \& Gullbring (1998) models do not display a hump around $5200 \AA$. When the excess continuum flux is divided by an assumed photospheric spectrum in order to compute the model veiling, a hump may appear in that spectral region. However, if the underlying photosphere corresponds to one of a G8V star, such as for DI Cep, no hump results from the Calvet \& Gullbring (1998) models. Indeed, in order to achieve a reasonable fit to the observed DI Cep veiling spectrum, one has to mask out the veiling measurements around $5200 \AA$.

Taking $M_{*}=0.5 M_{\odot}, R_{*}=2 R_{\odot}$, and the typical effective temperature for a G8V star, the best fit occurs for $\log \mathcal{F} \approx 12.0$ $\left(\mathcal{F} \approx\right.$ in units of erg $\mathrm{cm}^{-2} \mathrm{~s}^{-1}$ ) and a filling factor $f \approx 2.4 \%$. Models with different values of $\log \mathcal{F}$ are not able to simultaneously match both the veiling in the blue and in the red and the steep rise at $4500 \AA$. Models with $\log \mathcal{F}<12$ are too flat, while models with $\log \mathcal{F}>12$ are too steep. The Calvet \& Gullbring (1998) models are not too sensitive to the stellar mass and radius, such that differences between the true and assumed values for these parameters are not expected to change the values of the best fit $\log \mathcal{F}$ and $f$ appreciably. The derived filling factor agrees well with the 1-3\% estimate by Gómez de Castro \& Fernandez (1996), based on UV data, for a hot-spot covering the photosphere.

If we take the range of masses and radii, as previously estimated, and assume that the disk truncation radius is $5 R_{*}$, the free fall velocity ranges between 250 and $390 \mathrm{~km} \mathrm{~s}^{-1}$. By taking the accretion parameters $\mathcal{F}$ and $f$ that match the observations, we obtain a projected accretion rate $\dot{M} \cos (\theta) \sim$ $2.5 \times 10^{-7} M_{\odot} \mathrm{yr}^{-1}$ (Eq. (11) of Calvet \& Gullbring 1998; and see also Ardila \& Basri 2000). The average accretion rate in this range is slightly higher than the typical value for CTTS (see Johns-Krull \& Gafford 2002), but it agrees with the accretion rates determined independently by Gómez de Castro \& Lamzin (1999) and Johns-Krull et al. (2000) from UV data.

If one considers the underlying photospheric spectrum to be that of a K7V star, a Calvet \& Gullbring (1998) best model fit occurs for $\log \mathcal{F}=10.5$ and $f=0.06$. Such a model is shown in Fig. 1 as a dotted line. By using a K7V photosphere, one maximizes the strength of the $5200 \AA$ veiling spectrum hump that can be produced by current models. As is clear from Fig. 1, 
even then one cannot account for the observed hump, and the fit towards the blue gets significantly worse. In addition, the photospheric spectrum of DI Cep is well fit by a G8V template, showing no characteristics of a spectral type as late as K7.

\section{The veiling hump}

The DI Cep veiling in the wavelength range 5000-5400 $\AA$ is enhanced relative to the neighbouring regions by factors of up to 2 . This hump in the veiling spectrum is not explained by current models, and it does not seem to be particular to DI Cep. We identified similar features in the spectra of other CTTS (data to be discussed elsewhere) but not in WTTS or main sequence templates stars. This enhanced veiling region was first reported by Stempels \& Piskunov (2003, hereafter SP03), who analysed the photosphere and veiling spectrum of five T Tauri stars. By using synthetic template spectra, these authors find that, for the actively accreting stars, the recovered intensities of the veiling spectra around $5300 \AA$ have higher values than is expected from the continuous UV excess continuum. SP03 point out that such a feature can be reproduced neither by a single stellar continuum, or even a combination of them with different temperatures, nor by other continuum sources. In a more detailed analysis, SP03 consider three possible origins of the systematic errors: instrumental artifacts, systematic errors in the method of veiling measurements, and enhanced contribution from line emission. These three possibilities are dismissed as contributing towards the enhanced veiling feature. In particular, the last possibility is thoroughly tested by looking for systematic veiling overestimates due to clearly identified emission lines, but also by allowing for the presence of many unidentified weak emission lines that could mimic an extra veiling continuum emission or by considering a contribution from coronal emission lines.

SP03 further note that this enhanced veiling region is also visible, although not reported, in previous published investigations. They attribute this lack of reference to it to the scatter in the measurement and intrinsic veiling.

We argue that part of this feature may result from the broad absorption bands seen in the photospheric spectra of late type stars. Those bands have been used by Chelli (1999) to measure the veiling from low resolution spectra, when the individual photospheric absorption lines cannot be resolved. In high resolution spectra, normally obtained with echelle spectroscopy, the broad absorption spans several echelle orders. When those orders are continuum normalised, one effectively normalises them to a pseudo-continuum level that lies at the bottom of the broad feature and not to the true stellar continuum. The atomic photospheric lines in the normalised spectrum will therefore appear deeper than they really are. This effect is worse for the template stars, leading to overestimation of the veiling measured on the CTTS.

This argument is expressed by a schematic representation in Fig. 2. Panel (a) displays a fictitious echelle observation of a K5V template star (jagged solid line) built from the photospheric spectrum of a main sequence K5V star. The sloping continuum was implemented by multiplying the normalised $\mathrm{K} 5 \mathrm{~V}$ star spectrum by the dashed line and the broad absorption

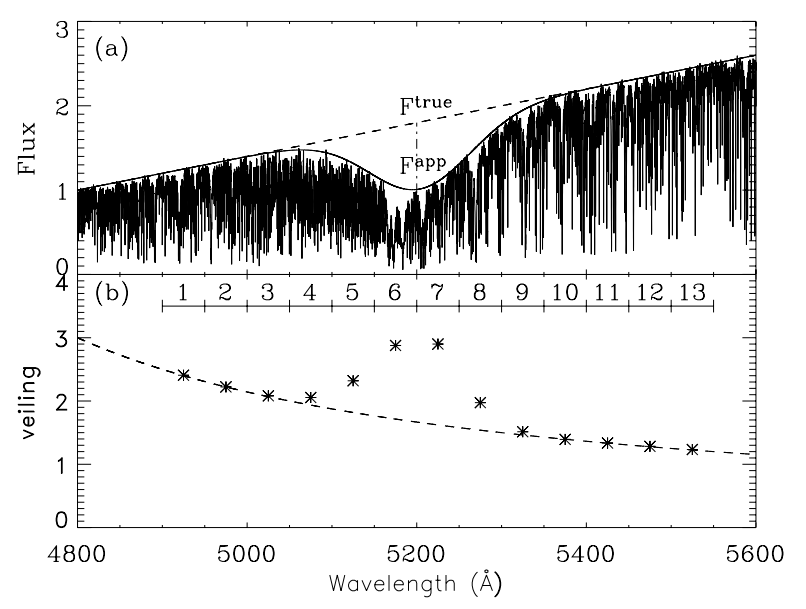

Fig. 2. a) Fictitious echelle observation of a $\mathrm{K} 5 \mathrm{~V}$ template star (jagged solid line). The "true" sloping continuum $\left(F^{\text {true }}\right)$ of the fictitious spectrum is represented by a dashed line, whereas the apparent continuum $F^{\text {app }}$ of the fictitious spectrum, as would be derived from an echelle observation, is represented by the smooth solid line. b) "True" veiling of an artificially veiled CTTS (dashed line) and veiling measurements (asterisks) determined after normalising each of the 13 echelle orders of the fictitious spectrum to the apparent continuum (see text for further details).

feature by dividing by a Gaussian centred at $5200 \AA$. The smooth solid line represents the apparent continuum level as it would be derived from the contiguous echelle orders labelled 1 to 13 .

The artificial spectrum of a CTTS with continuum excess emission (not shown here) was built simply by adding a constant flux of 3 (in the ad hoc flux units used in Fig. 2) to the spectrum shown on panel (a).

The veiling measurements shown as asterisks in Fig. 2, result from independently normalising the artificial template and CTTS spectra to the apparent continuum in each of the 13 echelle orders ( $50 \AA$ wide) indicated in Fig. 2 and then applying the usual procedure to compute the veiling. As clearly seen, the computed veiling is enhanced compared to the "real" veiling that was artificially imposed (dashed line on panel (b)).

One can understand the reason behind this enhancement by using the definition of veiling and expressing the absolute excess emission in terms of the veiling and continuum flux of the template star:

$F_{\lambda}^{\text {excess }}=r_{\lambda}^{\text {true }} \times F_{\lambda}^{\text {true }}=r_{\lambda}^{\text {app }} \times F_{\lambda}^{\text {app }}$

where $F_{\lambda}^{\text {true }}$ and $F_{\lambda}^{\text {app }}$ are the true and pseudo-photospheric continuum levels at a given wavelength, and $r_{\lambda}^{\text {true }}$ and $r_{\lambda}^{\text {app }}$ are the veiling obtained by considering either continua as reference.

By re-arranging the above equation, one can express the apparent veiling in terms of the real veiling and of the true to apparent continuum flux ratio:

$r_{\lambda}^{\text {app }}=r_{\lambda}^{\text {true }} \times \frac{F_{\lambda}^{\text {true }}}{F_{\lambda}^{\text {app }}}$.

This equation clearly shows that by underestimating the continuum level on the template star one overestimates the veiling. The increase factor does not depend upon the excess emission 


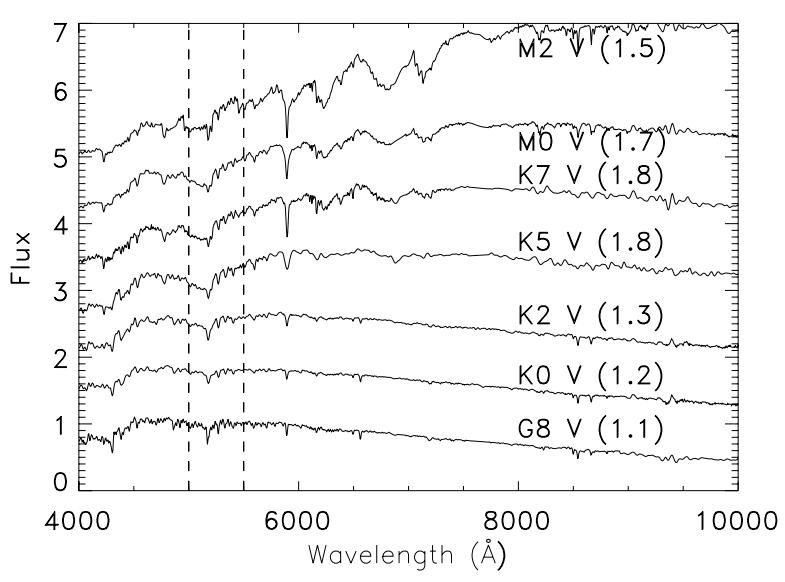

Fig. 3. Template spectra for spectral types G8V to M2V (Pickles 1985). The dashed lines mark the region of enhanced veiling seen in DI Cep and other CTTS. The ratio $F^{\text {true }} / F^{\text {app }}$ at the bottom of the absorption band, as defined in Fig. 2, is written within parenthesis. The spectra are shifted vertically for clarity.

on the CTTS but only on the estimate of the continuum level on the template star.

It should be pointed out that when there is no intrinsic excess emission, as in the case of WTTS, $r_{\lambda}^{\text {true }}$ is zero and, hence, $r_{\lambda}^{\text {app }}$ is also zero; i.e. no enhanced veiling will be detected. This is in agreement with observations: enhanced veiling emission around $5200 \AA$ is never observed on WTTS. An enhancement in veiling will be obtained for stars with excess continuum emission. Since the broad molecular features referred to above span several echelle orders, normalisation of the spectrum in each order will be done with respect to a pseudo-continuum level located below the real continuum level.

The relevance of this effect for veiling measurements can only be assessed by analysing the shape and depth of these absorption bands in real stars. In Fig. 3 we plot template spectra (from Pickles 1985) for main sequence stars with spectral types ranging from G8 to M2, typical of CTTS. There is a clear progression in the depth of the absorption band around $5200 \AA$ as the spectral type changes. In that figure, we write the ratio $F^{\text {true }} / F^{\text {app }}$ as defined in Fig. 2 by the spectral type of each spectrum. This ratio gives the maximum increase in veiling due to the absorption band. These values should be considered as lower limits, since to compute the $F^{\text {true }} / F^{\text {app }}$ ratios, we estimated $F^{\text {true }}$ by interpolating the continuum on either side of the absorption band. We expect that simple estimate to lie underneath the true continuum level across the band.

Clearly, the veiling enhancement is non-negligible for stars with spectral type later than $\mathrm{K} 2 \mathrm{~V}$, reaching almost a factor of 2 for $\mathrm{K} 5 \mathrm{~V}$ and $\mathrm{K} 7 \mathrm{~V}$ stars. On the other hand, the maximum enhancement expected for a G8 star is only around 1.1, which is not sufficient to explain the observed hump in the DI Cep veiling spectrum. For as hot a star as DI Cep, the increase in veiling due to a systematic error in the continuum normalisation, which in turn due to the presence of the molecular absorption at $5200 \AA$, is too small and cannot be responsible for the observed hump. In addition, the observed hump is significantly broader than expected from the discussed systematic effect, even in the case of later spectral type CTTS (see sample of veiling spectra
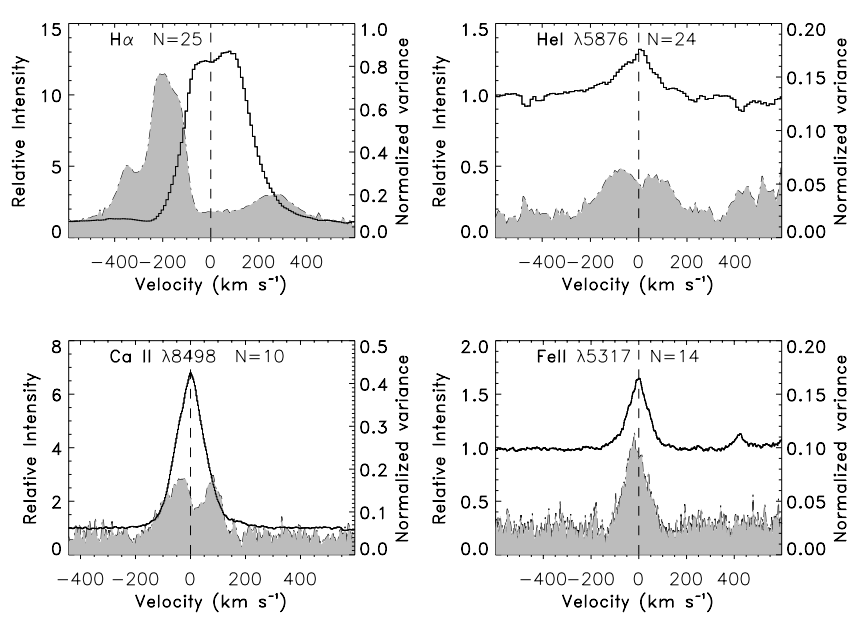

Fig. 4. Average and variance line profiles. Velocities are referred to stellar rest frame. The number of spectra used to compute the variance is given by $N$. The FeII profile corresponds to the residual profile, i.e. after removing the photospheric component. The hydrogen Paschen line Pa16 is located at velocity $+157 \mathrm{~km} \mathrm{~s}^{-1}$ relative to CaII.

presented by SP03), suggesting an unknown additional source of veiling.

Possible gravity effects on the depth and width of the molecular feature centred at $5200 \AA$ were looked into by comparing luminosity class $\mathrm{V}$ and III template spectra from Pickles (1985). The differences found were not significant enough to explain the origin of the observed hump in terms of the systematic effect discussed here.

\section{Spectral variability}

The profile variability of the strongest emission lines on the stellar rest frame, corrected to the average radial velocity of $-10.3 \mathrm{~km} \mathrm{~s}^{-1}$, are shown in Fig. 4, where the average profile (solid line) and the normalised variance profile (shaded area), as first defined by Johns \& Basri (1995), are overplotted. The CaII $\lambda 8498$ and FeII $\lambda 5317$ lines are representative of the CaII infrared triplet lines and FeII lines, respectively. The CaII $\lambda 8498$ is blended with the hydrogen Paschen line Pa16 (at $\lambda=8502.48 \AA$ or $+157 \mathrm{~km} \mathrm{~s}^{-1}$ relative to CaII $\lambda 8498$ rest velocity). Visual inspection of the average emission line profile does not provide a clear hint of the presence of Pa16.

The $\mathrm{H} \alpha$ line (and $\mathrm{H} \beta$ with similar variance profile) are the strongest variable lines in DI Cep's spectrum, with the blue wing varying the most. Variations in CaII and FeII lines occur mainly in their overall intensity, with only small changes in their shapes, even when the blue wing of $\mathrm{H} \alpha$ and $\mathrm{H} \beta$ is strongly varying due to the presence of absorption features.

The radial velocity derived by cross-correlating the various observed spectra with the template $\sigma$ Dra $(\mathrm{K} 0 \mathrm{~V})$ range from $-3 \mathrm{~km} \mathrm{~s}^{-1}$ to $-13 \mathrm{~km} \mathrm{~s}^{-1}$ and an average of $-10.3 \mathrm{~km} \mathrm{~s}^{-1}$. Given the average uncertainty in the measurements of $1.3 \mathrm{~km} \mathrm{~s}^{-1}$, the observed variations are probably real. We searched for periodicities in radial velocity, but we have not found any significant period. The lack of an adequate time sampling in the analysed data set may, however, hinder detection of any periodicity. New observations and further investigation are needed to clarify this 
point, in particular since the radial velocity variations may reflect real movement of the star and imply the presence of a companion object(s), or it may be an apparent effect of spots on the stellar photosphere (Petrov et al. 2001).

\section{Line flux vs. veiling}

The emission lines observed in the spectra of CTTS are expected to be formed, at least partially, in the accretion streams of gas within the magnetosphere. Correlations between emission line flux and veiling are therefore expected. Such correlations have been reported on various occasions. For example, for a sample of CTTS, Batalha et al. (1996) find a correlation between the flux in narrow and broad CaII line components and in veiling, whereas Bouvier et al. (2003) find time-delayed correlations between hydrogen and $\mathrm{He}$ I lines and veiling for the CTTS AA Tau.

The available studies tend to tackle line versus veiling correlations by considering the total line flux, or the line flux of a priori defined components as a whole. We propose a new tool to investigate the relationship between line emission/absorption and veiling. The idea is to correlate veiling not to the total line flux, but to the flux in discrete velocity bins across the emission line. This would reveal which parts of a given line profile do correlate with veiling and at what level. No a priori assumption has to be made regarding the line itself.

Before proceeding with the correlation computations, the line profile should be corrected for the presence of the (variable) underlying veiling continuum. The importance of this correction can be illustrated by the following simple example: since normalised to the continuum level an intrinsically nonvarying emission line on top of a varying underlying excess continuum emission, would have its profile anti-correlated with the veiling (see also discussions in Beristain et al. 2001; and Johns-Krull \& Basri 1997).

At any wavelength $\lambda$ bin, the corrected line flux $F_{\lambda}^{l}$ may be calculated as

$F_{\lambda}^{l}=\left(I_{\lambda}^{n}-1\right) \times\left(1+r_{\lambda}\right) \times F_{\lambda}^{\text {phot }}$

where $I_{\lambda}^{n}$ is the continuum normalised intensity, $F_{\lambda}^{\text {phot }}$ the continuum photospheric flux, and $r_{\lambda}$ the veiling factor. The veiling factor $r_{\lambda}$ can be taken as constant across a line profile.

Having performed the above correction, the set of time variable line fluxes for each wavelength (velocity) bin $F_{\lambda}^{l}(t)$ can be correlated with the set of time variable veiling factors $r_{\lambda}(t)$, resulting in a correlation coefficient profile across the line. We used the linear-correlation coefficient and estimated its significance following Bevington \& Robinson (1992).

We illustrate the use of this tool by applying it to the DI Cep data set presented here. In Fig. 5 we plot the zero lag correlation profiles, together with the time-averaged, veiling-corrected, emission line profiles for $\mathrm{H} \alpha$, HeI 15876 , CaII 18498 , and FeII $\lambda$ 5317. The data were not sampled well enough to try a lag analysis. In that figure, the left $y$-axis indicates relative line intensity, while the right $y$-axis (ranging from -1 to +1 ) indicates the value of correlation coefficient. The six horizontal dashed lines correspond to the correlations coefficients with
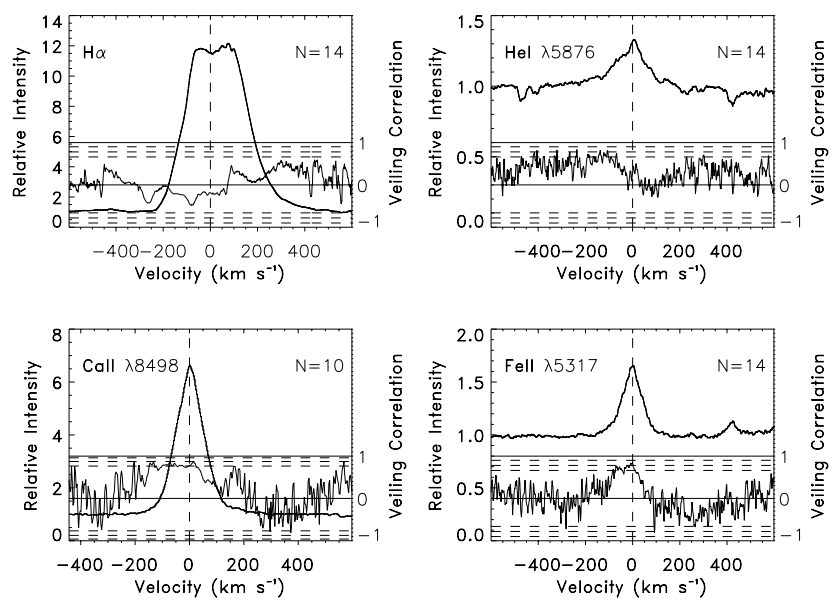

Fig. 5. "Line flux vs. veiling" correlation, superimposed on the average line profile (see explanation in the text). $N$ is the number of spectra used to compute the linear correlation.

99.9, 99.99, and 99.999\% confidence levels, respectively, as one moves from correlation zero to correlation \pm 1 . Significant correlations (anti-correlations) will intersect the top (bottom) three dashed lines.

Given the small number of spectra used in this analysis, we are not able to draw convincing conclusions. Nevertheless, we would like to spend a paragraph noting some aspects that are apparent from Fig. 5.

The $\mathrm{H} \alpha$ does not seem to be directly correlated with the veiling (the same applies to $\mathrm{H} \beta$ but the plot is not shown here). It should be noted that for AA Tau Bouvier et al. (2003) find a correlation between the $\mathrm{H} \alpha$ line flux and veiling only for a lag 1.08 days and, between $\mathrm{H} \beta$ and veiling, only for a lag of 0.44 days. Correlations are apparent at velocities blueward of the stellar rest velocity in the CaII, FeII, and maybe HeI lines. The peak of correlation that appears in the red wing of CaII $\lambda 8498$ is probably due to the hydrogen Paschen line Pa16 (not visible in the average line profile of Ca II due to its weakness), located at $+157 \mathrm{~km} \mathrm{~s}^{-1}$ relative to CaII $\lambda 8498$ rest velocity. Similar features appear at locations that correspond to blends between the other CaII infrared lines and hydrogen lines of the Paschen series. This agrees with the fact that higher members of the hydrogen Balmer series and lines of the Paschen and Brackett series are better tracers of accretion than $\mathrm{H} \alpha$ or $\mathrm{H} \beta$ (Edwards et al. 1994; Folha \& Emerson 2001). Somewhat surprising is the apparent lack of clear-cut correlations between the red wings of the analysed lines and veiling. This may be due simply to the poor sampling of the analysed data, but perhaps deserves further exploration by the acquisition of better data samples for this and other CTTS.

\section{Conclusions}

We have derived the optical veiling spectrum of DI Cep and shown that, while it agrees globally with what is expected from accretion shock models, it also displays a clear hump located at around $5200 \AA$, which is not predicted by models. Such a feature has been independently identified in the spectra of other CTTS by Stempels \& Piskunov (2003). We examined in 
what measure this hump may be caused by a systematic effect, namely the presence of a molecular photospheric absorption band in the spectra of late type main sequence stars, used as templates for the veiling measurements. While such an affect may be important for stars with spectral types later than $\mathrm{K} 2$, it certainly does not explain the hump identified here for the G8 star DI Cep.

We propose a new diagnostic tool for analysing the relationship between line emission/absorption and veiling and illustrate its use with DI Cep data. The small number of available spectra prevented us from drawing strong conclusions. Nevertheless, it seems clear that positive correlations with veiling are present for: 1) at least the emission blueward of the stellar rest velocity in the CaII infrared triplet lines and FeII lines; 2) the higher members of the hydrogen Paschen series that appear blended with the CaII infrared triplet lines.

We measured DI Cep's $v \sin i$ to better than $10 \%$ and used it, together with data found in the literature, to constrain DI Cep's inclination to the line-of-sight. We concluded that DI Cep is observed nearly edge on. And we found that DI Cep's radial velocity varies with time. Given our average uncertainty of $1.3 \mathrm{~km} \mathrm{~s}^{-1}$, we found variations in the range -3 to $-13 \mathrm{~km} \mathrm{~s}^{-1}$. No periodicities were found from our time series, most probably due to the poor time sampling of the data set. DI Cep should next be thoroughly investigated for radial velocity variations.

Acknowledgements. The authors wish to thank Dr. Nuria Calvet for kindly allowing them to use outputs of the Calvet \& Gullbring (1998) accretion shock models. Part of this work was financially supported by projects POCTI/1999/FIS/34549 and POCTI/CTE-AST/55691/2004, approved by FCT and POCTI, with funds from the European Union programme FEDER and in part by the European Union Marie Curie Actions - Human Resource and Mobility within the JETSET (Jet Simulations, Experiments and Theory) network under contract MRTN-CT-2004-005592. DFMF acknowledges financial support by FCT from the "Subprograma Ciência e Tecnologia do $3^{\circ}$ Quadro Comunitário de Apoio". P.P.P. acknowledges support from a CAUP grant through "Financiamento Plurianual" and financial support from INTAS grant 03-51-6311.

\section{References}

Ardila, D. R., \& Basri, G. 2000, ApJ, 539, 834

Baranne, A., Queloz, D., Mayor, M., et al. 1996, A\&AS, 119, 373

Bastian, U., \& Mundt, R. 1979, A\&A, 78, 181
Batalha, C. C., Stout-Batalha, N. M., Basri, G., \& Terra, M. A. O. 1996, ApJS, 103, 211

Bertout, C., Basri, G., \& Bouvier, J. 1988, ApJ, 330, 350

Beristain, G., Edwards, S., \& Kwan, J. 2001, AJ, 551, 1037

Bevington, P. R., \& Robinson, D. K. 1992, Data Reduction and Error Analysis for the Physical Sciences (McGraw-Hill)

Bouvier, J., Grankin, K. M., Alencar, S. H. P., et al. 2003, A\&A, 409, 169

Calvet, N., \& Gullbring, E. 1998, ApJ, 509, 802

Camenzind, M. 1990, Rev. Mod. Astron., 3, 234

Chelli, A. 1999, A\&A, L65

D’Antona, F., \& Mazzitelli, I. 1994, ApJS, 90, 467

Edwards, S., Hartigan, P., Ghandour, L., \& Andrulis, C. 1994, AJ, 108, 1056

Folha, D. F. M., \& Emerson, J. P. 2001, A\&A, 365, 90

Gahm, G. F., Gershberg, R. E., Petrov, P. P., et al. 1977, PZSSR, 20, 381

Gahm, G. F., \& Petrov, P. P. 1983, in Activity in red-dwarfs stars, ed. P. B. Byrne, \& M. Rodonó (Dordrecht: Reidel), 497

Gómez de Castro, A. I., \& Fernández, M. 1996, MNRAS, 283, 55

Gómez de Castro, A. I., \& Lamzin, S. A. 1999, MNRAS, 304, 41

Ilyin, I. V. 2000, Ph.D. Thesis, University of Oulu

Hamann, F., \& Persson, S. E. 1992, ApJ, 394, 628

Hartigan, P., Hartmann, L., Kenyon, S., Hewett, R., \& Stauffer, J. 1989, ApJS, 70, 899

Herbig, G. H. 1970, Mem. Soc. R. Sci. Liege, 19, 13

Herbig G. H., \& Bell K. 1988, Lick Obs. Bull., 1111

Hessman, F. V., \& Guenther, E. W. 1997, A\&A, 321, 497

Johns, C. M., \& Basri, G. 1995, AJ, 109, 2800

Johns-Krull, C. M., Valenti, J. A., \& Linsky, J. L. 2000, ApJ, 539, 815

Johns-Krull, C. M., \& Basri, G. 1997, ApJ, 474, 433

Johns-Krull, C. M., \& Gafford, A. D. 2002, ApJ, 573, 685

Kholopov, P. N. 1959, Soviet Astron., 3, 291

Kolotilov, E. A., Metlov, V. G., Metlova, N. V., \& Petrov, P. P. 2004, Astron. Astrophys. Trans., 23, 185

Königl, A. 1991, ApJ, 370, L39

Lynden-Bell, D., \& Pringle, J. E. 1974, MNRAS, 168, 603

Muzerolle, J., Calvet, N., \& Hartmann, L. 2001, ApJ, 550, 944

Palla, F., \& Stahler, S., ApJ, 525, 772

Petrov, P. P. 1972, Izv. Krymsk. Astrofiz. Obs., 46, 25

Petrov, P. P., Gahm, G. F., Gameiro, J. F., et al. 2001, A\&A, 369, 993

Pickles, A. J. 1985, 1985, ApJS, 59, 33

Shu, F., Najita, J., Ostriker, E., et al. 1994, ApJ, 429, 781

Stempels, H. C., \& Piskunov, N. 2003, A\&A, 408, 693

Tody, D. 1993, IRAF in the Nineties in Astronomical Data Analysis Software and Systems II, ed. R. J. Hanisch, R. J. V. Brissenden, \& J. Barnes, ASP Conf. Ser., 52, 173

Yu, K. 1986, Astron. Tsirk, 1459, 5 